\title{
Beam-Splitting in plasmonic multimode waveguides based on the self-imaging effect
}

\author{
A. Edelmann, S. Helfert, and J. Jahns \\ FernUniversität in Hagen, Universitätsstr. 27/PRG, 58084 Hagen, Germany \\ Correspondence to: A. Edelmann (andre.edelmann@fernuni-hagen.de)
}

\begin{abstract}
A plasmonic $1 \times 2$ beamsplitter based on the selfimaging effect is analysed. The simulations were performed by a 3-D full-vectorial numerical calculation. We discuss the coupling efficiency depending on structural parameters and on the attenuation.
\end{abstract}

\section{Introduction}

The field of plasmonics deals with electromagnetic waves which propagate at the interface between a dielectric and metallic medium. These electromagnetic waves are called surface plasmon polaritons (SPPs). The SPPs couple with the free electrons of the metal excited by an evanescent field, resulting e.g. from a prism or grating. The incident light has to be TM polarised to obtain an electric component normal to the interface. The SPPs have the same frequency with the incident light but due to the larger k-vektor a smaller wavelength. A profound knowledge of the physical understanding of SPPs is given, e.g., in Raether (1988). Plasmonics offer the potential towards higher integration densities for optical circuits by combining the high capacity in photonics and the miniaturisation technologies in electronics. One of the challenges in plasmonics refers to the attenuation of the propagating surface waves caused by the metal. Here propagation lengths of several tens of micrometers are achievable. The attenuation depends on various parameters, e.g., the wavelength or the dielectric media and can be generally reduced by accepting a higher extension of the field in the dielectric. More detailed information about the potential of plasmonics are specified in Ozbay (2006); Maier and Atwater (2005).

Beam splitters are an important functionality for integrated optical circuits. Several concepts in plasmonics have been developed, e.g., Y-shaped splitters (Han et al., 2010), Tshaped splitters (Veronis and Fan, 2005) and splitters relates to the multimode interference (MMI) (Han and He , 2007; Yuan et al., 2009). Here, we focus on beam splitters re-

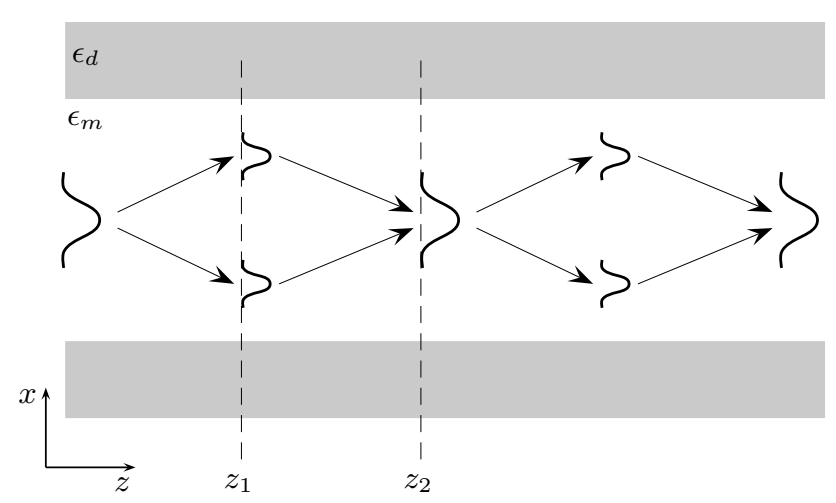

Fig. 1. Self-imaging effect in a multimode waveguide. At position $z_{1}$ the field is split and at the position $z_{2}$ the input field is reproduced.

garding the MMI. These are based on the self-imaging phenomenon. Our intention is to find out detailed information about the characteristic regarding the beam splitter by varying several structural parameters.

This article is organised as follows: in Sect. 2 we show how the beam splitting can be achieved in multimode waveguides; in Sect. 3 we describe the numerical algorithm and the model of the structure; in Sect. 4 we present and discuss our findings and in Sect. 5 we conclude our work.

\section{Self-imaging based beam splitting}

The self-imaging effect in dielectric and plasmonic waveguides leads to periodic repetition of the injected field profile (Soldano and Pennings, 1995; Edelmann et al., 2010). Fig. 1 shows the principle. One can find a 2-D plasmonic waveguide with the metallic core at the permittivity $\epsilon_{\mathrm{m}}$ in the center surrounded by a dielectric media with the permittivity $\epsilon_{\mathrm{d}}$. As indicated in the figure, we inject a field with narrow 
shape (no eigenmode) on the left into the waveguide. The input field propagates in $z$-direction. As shown, we find doubled self-images of the field at the position $z_{1}$ and an image of the input field distribution at the position $z_{2}$. The selfimaging phenomenon occurs because of interference of the excited modes in the waveguide. Note: in Fig. 1 we have shown the self-imaging effect for the special case of a symmetric field profile which is injected at the center of the waveguide. The periodicity of the self-imaging is determined by $z_{2}=3 / 8 \lambda_{0} / \Delta \bar{\beta}$. Here, $\Delta \bar{\beta}$ means the difference in normalized propagation constants of the two lowest-order modes. More specified information can be found in (Soldano and Pennings, 1995).

Based on the self-imaging effect in multimode waveguides, one can realise a beam splitters by out-coupling the doubled images via two separated waveguides at the position $z_{1}$. Approaches for that kind of beam splitting in plasmonic waveguides are studied in e.g., Han and $\mathrm{He}$ (2007); Yuan et al. (2009).

\section{Numerical modelling of the structure}

Our results are gained with full vectorial calculations using the Method of Lines (MoL). Here we restrict ourselves to a brief summary, detailed information can found in e.g., Pregla and Pascher (1989); Pregla (2008). The MoL is an eigenmode propagation method. The cross-section of the considered structure is discretized with finite differences (FD). The eigenmodes are obtained by combining the FD expressions in an operator matrix. Then the eigenvalues and eigenvectors of this operator matrix represent the propagation constant and the field distribution of the eigenmodes. The further calculations are done analytically.

Figure 2a shows the cross-section of the plasmonic waveguide which is used in our studies. One can find the metallic layer in the center surrounded by dielectric medium. The computation window has the dimensions $w_{x}=4.15 \mu \mathrm{m}$ and $w_{y}=0.875 \mu \mathrm{m}$ with the discretisation distances $h_{x}=$ $0.05 \mu \mathrm{m}$ and $h_{y}=0.0125 \mu \mathrm{m}$. The calculations are carried out at the wavelength $\lambda=0.633 \mu \mathrm{m}$. For the metallic layer width we choose $w_{\mathrm{m}}=3 \mu \mathrm{m}$ and for the thickness $t_{\mathrm{m}}=0.125 \mu \mathrm{m}$. For the metal we use gold with the permittivity $\epsilon_{\mathrm{m}}=-14.2035-j 0.7621$ (obtain from the Drude-model (Palik, 1985) with the time dependence according to $e^{+j \omega t}$ ) and for the dielectric we choose $\epsilon_{\mathrm{d}}=4$. The calculated field profile of the first fundamental mode is also plotted in Fig. 2a. As is known, the maxima of the field are located at the interfaces in $y$-direction and exponentially decay into the dielectric media and the metal. The longitudinal section at the metallic/dielectric interface of the analysed beam splitter is shown in Fig. 2b. The geometrical parameters of the structure are: width of the input waveguide $w_{\mathrm{i}}=1.1 \mu \mathrm{m}$; length of the input waveguide $z_{\mathrm{i}}=1.0 \mu \mathrm{m}$; width of the middle multimode waveguide $w_{\mathrm{m}}=3.0 \mu \mathrm{m}$; length of the middle wave-

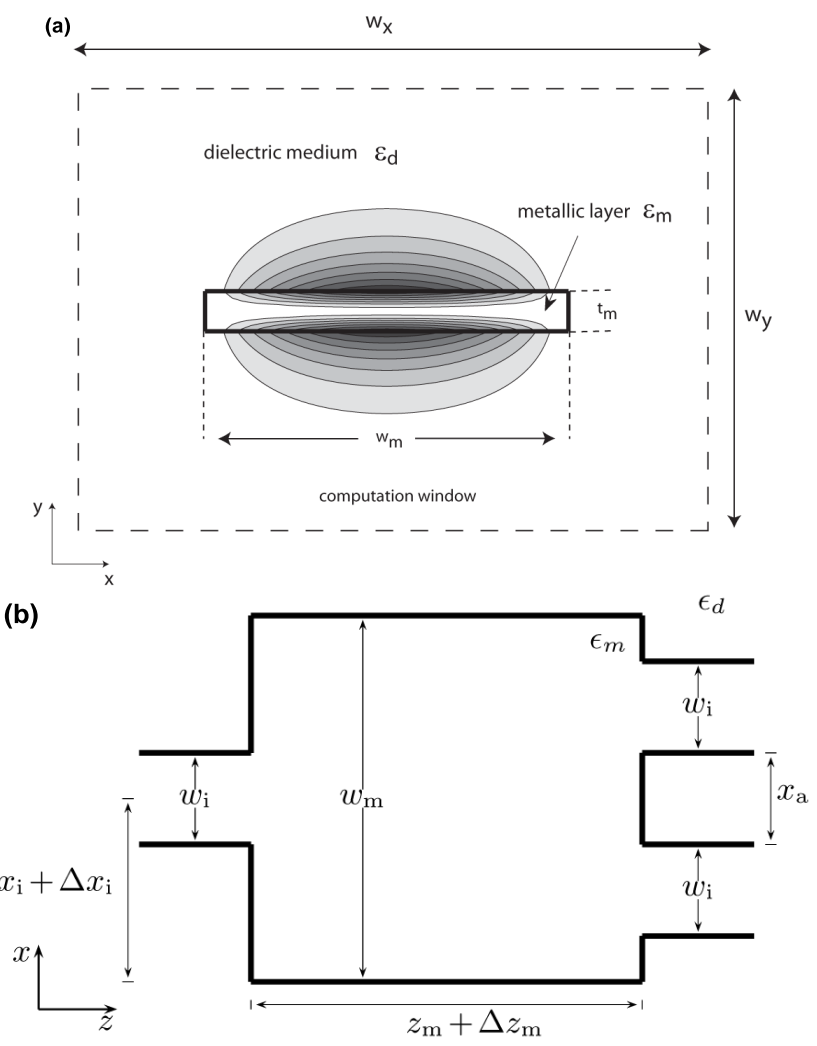

Fig. 2. (a) Cross-section of the computed structure and field profile of the first fundamental mode. (b) Longitudinal section of the analysed beam splitter.

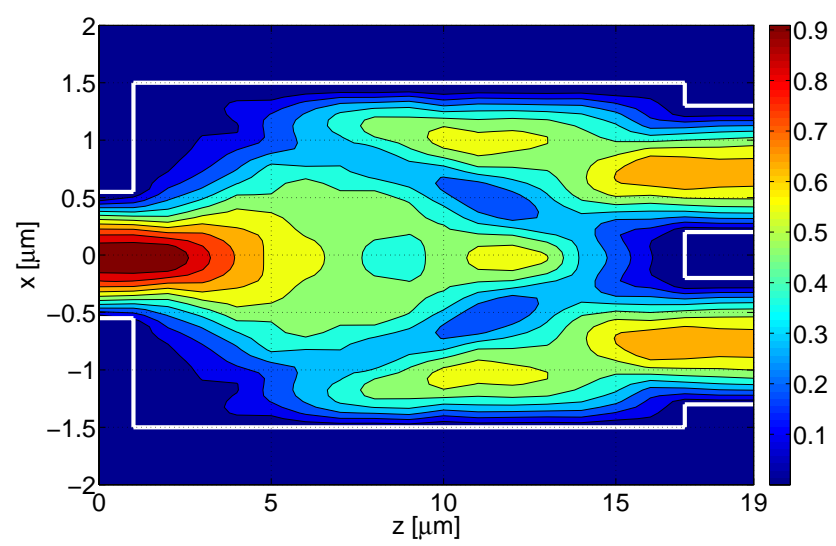

Fig. 3. Calculated field profile of the plasmonic beam splitter shown in Fig. 2 (losses are neglected).

guide $z_{\mathrm{m}}=16 \mu \mathrm{m}$. The output waveguides have the same dimensions as the input waveguide. The output waveguides are symmetrically placed around the centre of the structure with distance between them of $x_{\mathrm{a}}=0.4 \mu \mathrm{m}$.

In the following we examine the influence of a few geometrical parameters. 

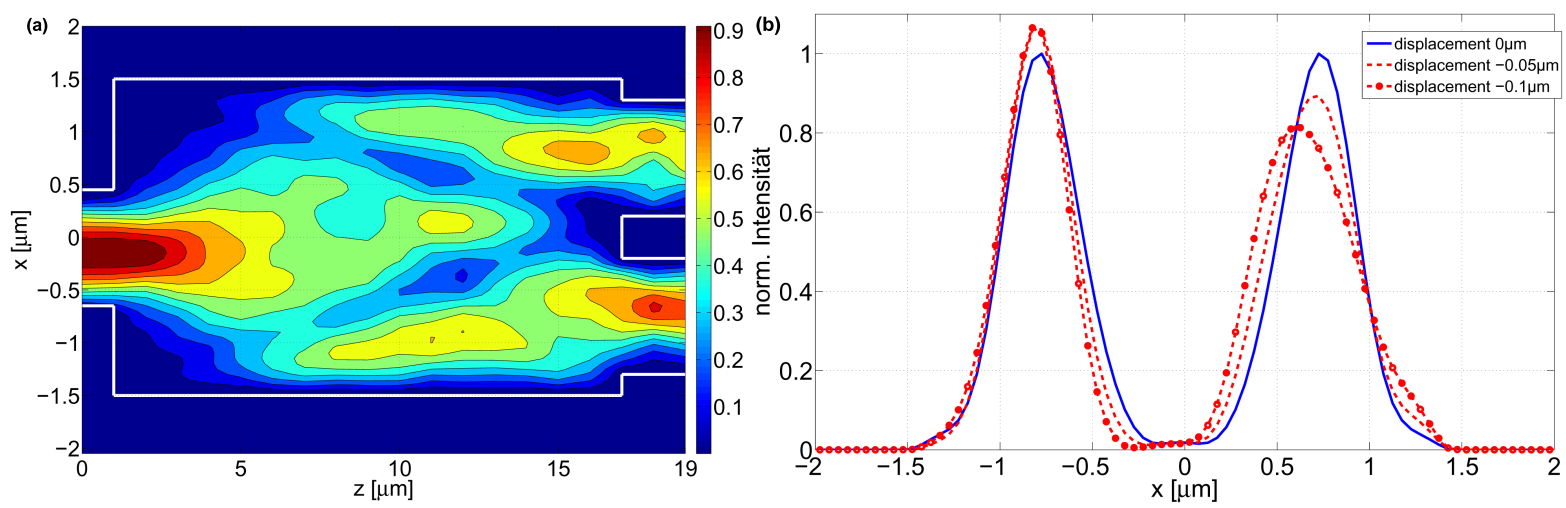

Fig. 4. (a) Calculated field (3-D) at the metallic/dielectric interface. The input waveguide is shifted by $\Delta x_{\mathrm{i}}=-0.1 \mu \mathrm{m}$ to the central position $x_{\mathrm{i}}$. (b) Related intensity profile at the output waveguide for for the shifts $\Delta x_{\mathrm{i}}=-0.05 \mu \mathrm{m}$ and $\Delta x_{\mathrm{i}}=-0.1 \mu \mathrm{m}$.
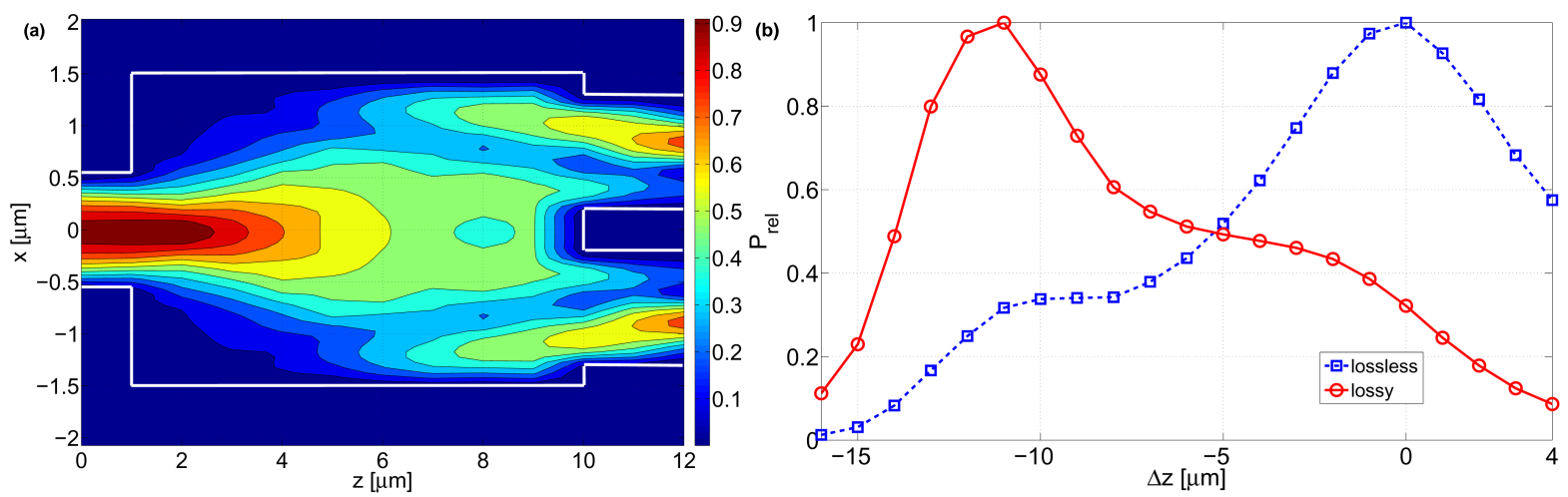

Fig. 5. Calculated field (3-D) at the metallic/dielectric interface. The middle waveguide is shorted by $\Delta z_{\mathrm{m}}=-6 \mu \mathrm{m}$ relative to the optimal position $z_{\mathrm{m}}$ (losses are neglected). (b) Peak power in the output waveguides normalised to peak power at the optimal coupling position.

\section{Results}

In this section we present the numerical results. Figure 3 shows the calculated field profile in the dielectric/metallic interface for the structure shown in Fig. 2. The fundamental mode of the input waveguide is injected into the middle multimode waveguide. Within the middle waveguide the field is symmetrically split due to the self-imaging effect into two separate images which couple into the output waveguides. Below we discuss the influence of the position of the input waveguide in dependance of $\Delta x_{\mathrm{i}}$ and the influence of the length of the middle waveguide by varying $\Delta z_{\mathrm{m}}$.

\subsection{Position of the input waveguide}

Figure 4a shows the field distribution when the input waveguide is shifted at $\Delta x_{\mathrm{i}}=-0.1 \mu \mathrm{m}$. The field distribution in the middle waveguide and in the output waveguides is no longer symmetric. In Fig. $4 \mathrm{~b}$ the intensity profile in the output section is plotted. The continuous curve relates to the unshifted case. The dashed lines relate to a shift in negative di- rection with $\Delta x_{\mathrm{i}}=-0.05 \mu \mathrm{m}$ and $\Delta x_{\mathrm{i}}=-0.1 \mu \mathrm{m}$. If we increase the displacement of the input waveguide the intensity for upper output waveguide decreases, whereas the intensity in the lower output waveguide remains almost constant. The source of that characteristic results from the unsymmetric interference of the excited modes at the out-coupling position. Note: Due to the symmetric structure we find analogue results if we shift the input waveguide in positive direction.

\subsection{Length of the middle waveguide}

Next we analysed the influence of the length of the middle waveguide $z_{\mathrm{m}}$. In Fig. 5a the field distribution for $\Delta z_{\mathrm{m}}=$ $-6 \mu \mathrm{m}$ (i.e. the length of the middle waveguide is reduced) is plotted. If we compare this figure with Fig. 3 we find significant deviations of the field distribution due to the worse coupling position. To quantify the relation between the coupling efficiency and the length of the center waveguide we determine the maximum of the field intensity in the output section (as shown in Fig. 4b) as function of the deviation of 
the center waveguide $\Delta z_{\mathrm{m}}$. The result can be seen in Fig. $5 \mathrm{~b}$. The dashed line shows the behaviour for neglected losses whereas the continuous line takes the losses of the metal into account. Assuming lossless metal, the maximum intensity is located at $\Delta z_{\mathrm{m}}=0$ and decays by shifting $\Delta z_{\mathrm{m}}$ in positive or negative direction. At $\Delta z_{\mathrm{m}}=0$ the best coupling position appears, where we have the doubling image of the input signal. If we take the losses into account the maximum of the intensity is given for a shift of $\Delta z_{\mathrm{m}}=-11 \mu \mathrm{m}$. Here the coupling of the field to the output waveguides is inferior to $\Delta z_{\mathrm{m}}=0$ but due to the reduced attenuated amplitude of the field we obtain in sum a higher intensity in the output waveguides.

\section{Conclusions}

We have analysed a plasmonic beam splitter based on the self-imaging effect. The field distribution of the beam splitter was calculated by 3-D full vectorial calculations using the MoL. Particular we studied the influence of the lateral position of the input waveguide and the length of the middle waveguide on the coupling efficiency. We found that the ratio of the field at the output waveguides can be modified by geometrical parameters (symmetrically as well as asymmetrically). In particular we have considered the losses of the metal regarding the coupling efficiency. It is found that the highest coupling efficiency does not occur at the location of the doubled self-imaging, but at an earlier position, due to the propagation losses.

Acknowledgements. We thank Christopher Alain Jones for reading this manuscript.

\section{References}

Edelmann, A. G., Helfert, S. F., and Jahns, J.: Analysis of the selfimaging effect in plasmonic multimode waveguides, Appl. Opt., 49, A1-A10, 2010.

Han, Z. and He, S.: Multimode interference effect in plasmonic subwavelength waveguides and ultra-compact power splitter, Opt. Comm., 278, 199-203, 2007.

Maier, S. A., Atwater, H. A.: Plasmonics: Localization and guiding of electromagnetic energy in metal/dielectric stuctures, J. Appl. Phys., 98, 011101:1-10, 2005.

Han, Z., Elezzabi, A. Y., and Van, V.: Wideband Y-splitter and aperture-assisted coupler based on sub-diffraction confined plasmonic slot waveguides, Appl. Phys. Lett., 96, 131106:1-3, 2010

Ozbay, E.: Plasmonics: Merging photonics and electronics at nanoscale dimensions, Science, 311, 189-193, 2006.

Palik, E. D.: Handbook of Optical Constants of Solids, Academic Press, London, UK, 1985.

Pregla, R. and Pascher, W.: The method of lines, in: Numerical Techniques for Microwave and Millimeter Wave Passive Structures, edited by: Itoh, T., J. Wiley Publ., New York, USA, 381446, 1989.

Pregla, R.: Analysis of electromagnetic Fields and Waves: The Method of Lines, John Wiley \& Sons, Ltd., West Sussex, England, 2008.

Raether, H.: Surface plasmons on smooth and rough surfaces and on gratings, Springer, Berlin, Germany, 1988.

Soldano, L. B. and Pennings, E. C. M.: Optical multi-mode interference device based on self-imaging: principles and applications, J. Lightwave Technol., 13, 615-627, 1995.

Veronis, G. and Fan, S.: Bends and splitters in metal-dielectricmetal subwavelength plasmonic waveguides, Appl. Phys. Lett., 87, 131102:1-3, 2005.

Yuan, G., Wang, P., Lu, Y., and Ming, H.: Multimode interference splitter based on dielectric-loaded surface plasmon polariton waveguides, Opt. Express, 19, 12594-12600, 2009. 